\title{
Noise Analysis for Sampling Mixers Using Stochastic Differential Equations
}

\author{
Wei Yu and Bosco H. Leung, Senior Member, IEEE
}

\begin{abstract}
We analyze three different sources of noise in a sampling mixer working at radio frequency $(\mathrm{RF})$ or intermediate frequency. External RF and intrinsic noise are analyzed using conventional frequency domain methods. External local oscillator (LO) noise is analyzed in time domain by solving a stochastic differential equation. We are able to take into account the timevarying aspect of the LO noise coupling, and show that LO noise becomes important at high frequencies. Analytical expression for LO noise is obtained, which can be used to guide mixer design. LO noise is in additional to LO jitter, which should be combined with noise analysis to give a complete picture.
\end{abstract}

Index Terms - Intermediate frequency (IF), noise, radio frequency (RF), sampling mixer, stochastic differential equation (SDE).

\section{INTRODUCTION}

$\mathbf{T}$ THE RECENT surge of interests in wireless digital communication has created a demand for implementing compact low-power low-noise wireless receivers in CMOS technology. In the receiver chain, it is desirable to perform front-end operations such, as mixing and analog/digital (A/D) conversion as early as possible, ideally on a radio frequency (RF) signal directly [1]-[3]. However, early conversion also puts stringent performance requirements on the $\mathrm{A} / \mathrm{D}$ converter, which has to operate at the much higher RF frequency. At high frequency, noise becomes a significant problem. Theoretical and experimental results show that one of the key elements in $\mathrm{A} / \mathrm{D}$ conversion which limits noise performance is the front-end track-and-hold sampling mixer, which simultaneously performs sampling and mixing. In this paper, we will concentrate on the noise analysis for the sampling mixer.

Noise enters a circuit via various paths. In a sampling mixer, noise may come from the external RF port, the switching local oscillator (LO) port, or from within the mixer itself. All three sources must be taken into account for a complete noise analysis. Circuit noise analysis is traditionally done in frequency domain. Noise sources are assumed to be white with a flat power spectral density. This approach is simple

Manuscript received September 24, 1997; revised February 19, 1999. This work was supported by the Department of Electrical and Computer Engineering, University of Waterloo, and by the National Science and Engineering Research Council (NSERC) of Canada. This paper was recommended by Associate Editor J. B. Dias.

W. Yu was with the Department of Electrical and Computer Engineering, University of Waterloo, ON N2L 3G1 Canada. He is now with the Information System Laboratory, Department of Electrical Engineering, Stanford University, Stanford, CA 94305 USA.

B. H. Leung is with the Department of Electrical and Computer Engineering, University of Waterloo, ON N2L 3G1, Canada.

Publisher Item Identifier S 1057-7130(99)04876-4.

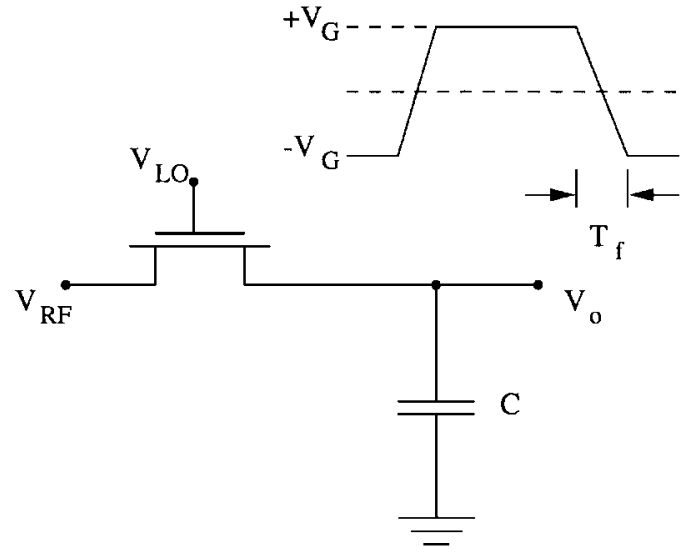

Fig. 1. Track-and-hold sampling mixer.

and effective in cases where the circuit is linear and timeinvariant, or where it can be approximated as such. In the sampling mixer, this approach is applicable to the analysis of intrinsic and external RF noise, but not to LO noise. In the case of LO noise, the system is neither linear nor time-invariant due to the switching nature of the LO waveform. A rigorous analysis of LO noise is therefore a challenging problem.

In this paper, we will use the traditional frequency domain method to solve for RF and intrinsic noise. For LO noise, we will advocate a time domain approach based on solving a stochastic differential equation (SDE). The method of SDE's in circuit noise analysis was used in [4] from a circuit simulation point of view. Their approach is based on the linearization of SDE's about its simulated deterministic trajectory. In this paper, we will use a different approach from which an analytical solution to the SDE will be obtained. The analytical solution will take into account the circuit time-varying nature, and we will show that LO noise becomes important at high frequency.

The rest of the paper is organized as follows. Section II classifies different sources of noise in the mixer, and reviews the conventional method for solving intrinsic and external RF noise. Section III derives the ordinary differential equation governing the noise variance process, and solves them in the sampled data domain. We summarize and conclude in Section IV.

\section{NOISE IN A SAMPLING MiXER}

\section{A. Sampling Mixer}

We consider a sampling mixer which consists of a MOS transistor followed by a sampling capacitor (Fig. 1). The 


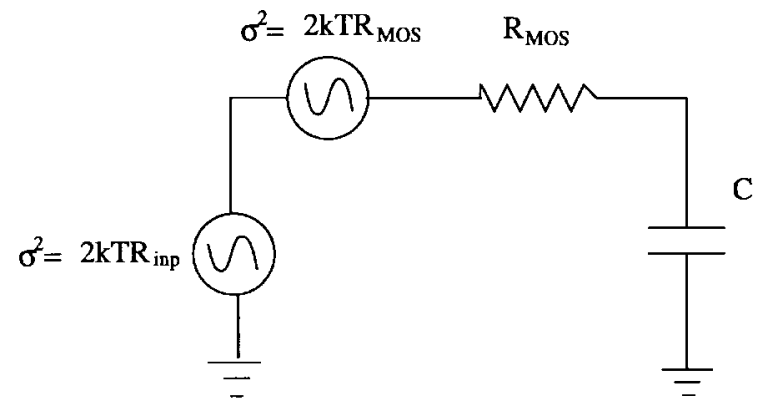

Fig. 2. Low-pass filtered noise.

input $V_{\mathrm{RF}}$ is the RF signal or its mixed-down version at the intermediate frequency (IF). The switch is designed to have the time constant formed by the MOS and the capacitor very small, so that the output $V_{O}$ tracks the input when the LO voltage applied at the gate is high. When LO goes low, the output $V_{o}$ is sampled and held on the capacitor. This circuit not only serves as a track-and-hold front-end to A/D conversion, but also acts as a mixer that mixes down RF or IF signals to the baseband. Notice that the output should be considered as sampled data, since the output is to be converted to digital domain immediately.

There are three potential noise sources in the circuit. There is noise from the RF port, there is device intrinsic noise from the MOS switch, and there is noise from the LO port, all of which can potentially be coupled into the output. Each of the three factors must be analyzed separately.

\section{B. External and Intrinsic Noise}

First let us consider the sampling mixer with its gate voltage fixed at high. This corresponds to a continuous time-invariant nonmixing operation, where the MOS switch and the capacitor form a lower pass filter with filter bandwidth approximately equal to $g / C$, where $g=K\left(V_{G}-V_{t}\right)$ is the MOS onconductance. This neglects the square law nonlinearity in the MOS device equation, which is justifiable because the nonlinearity must be small by design to avoid harmonic and intermodulation distortion. With the assumption of linear time-invariant operation, we can now use frequency domain methods to analyze noise from the external and the intrinsic sources.

Let us suppose that both input and intrinsic noise is white with combined two-sided power spectral density at $\sigma^{2}=$ $2 k T\left(R_{\mathrm{inp}}+R_{\mathrm{mos}}\right)$, where $R_{\mathrm{inp}}$ and $R_{\mathrm{mos}}$ are the equivalent input noise resistor and intrinsic noise resistor, respectively. Then, the noise at the output will have the power spectral density shaped in a low-pass fashion with $-3-\mathrm{dB}$ frequency equal to the bandwidth of the low-pass filter (Fig. 2). The total noise power at the output can be calculated by summing the area under the power spectral density curve

$$
E\left[N_{t}^{2}\right]=\frac{k T}{C}\left(1+\frac{R_{\mathrm{inp}}}{R_{\mathrm{mos}}}\right)
$$

where $N_{t}$ is the output noise process. The $k T / C$ part is due to intrinsic noise, and it is independent of the MOS on-

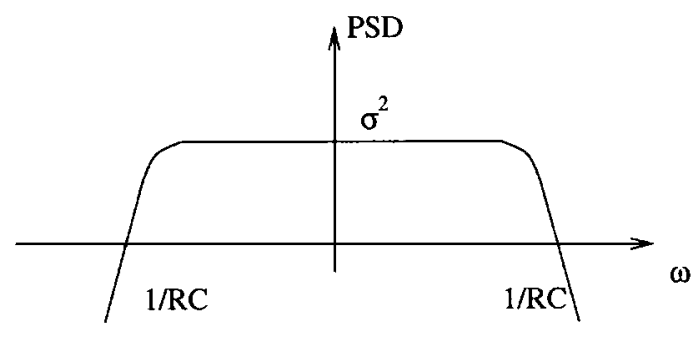

conductance. The second part is due to external noise. The noise variance calculated above is independent of sampling.

The low-pass shape of the power spectral density also gives us the second-order statistics of the output-random process. In time domain, this means that the correlation between consecutive samples in the output process decreases exponentially with its time constant equal to that formed by the MOS and the capacitor

$$
E\left[Y_{t} Y_{s}\right]=\frac{k T}{C}\left(1+\frac{R_{\mathrm{inp}}}{R_{\mathrm{mos}}}\right) e^{-|t-s| / R_{\mathrm{mos}} C} .
$$

Now we consider the effect of sampling. From (2), we see that the correlation between samples decreases exponentially. As noted before, the time constant of the switch is designed to be much smaller than the period of the input signal to allow accurate tracking. So, the time constant must also be much smaller than the switch period, which means that consecutive samples are mostly uncorrelated. Therefore, in a sampled data domain, discrete output samples can be considered independently identically distributed (i.i.d.), whose discrete power spectral density is folded into the frequency band from zero up to $f_{s} / 2$, where $f_{s}$ is the sampling frequency. Therefore, if the signal has bandwidth $f_{b}$, the total noise power inside the signal band is

$$
\frac{k T}{C}\left(1+\frac{R_{\mathrm{inp}}}{R_{\mathrm{mos}}}\right) \cdot \frac{2 f_{b}}{f_{s}} .
$$

From the above equation, it can be seen that in the case of subsampling, although noise variance stays the same, the amount of noise in the signal band increases by the subsampling ratio. Also, we note that in the case where the sampling edge has a nonzero fall time, (3) still serves as an upper bound because the MOS resistance increases as the gate voltage falls; consequently, the total noise power in this case will be smaller than that predicted by (3).

We have assumed in the previous derivation that the intrinsic noise is white. The white noise assumption is only true for thermal noise, but not for $1 / f$ noise, which is also present in MOS devices. Because $1 / f$ noise is low pass, it is unaffected by the presence of the capacitor. The effect of the sampling is simply to move its power spectral density by $f_{s}$. Since we are only interested in noise in the signal band, and the mixed down-signal frequency is usually much higher than the frequency where $1 / f$ noise is large, we can safely neglect $1 / f$ noise in the analysis. 


\section{LO Noise}

There are two distinct mechanisms by which noise at LO can contribute to total noise at the output. First, due to noise at the LO port, the time at which sampling occurs is random. This is what we refer to as LO jitter, and it is intrinsic to the sampling operation. LO jitter has been analyzed in the literature using discrete stochastic process techniques. For example, [5] analyzed jitter power spectral density for CMOS ring oscillators, and [6] showed how jitter power is related to noise power at the output. The second mechanism is due to the fact that noise at the LO port causes the MOS conductance to fluctuate, hence producing noise at the output. We call this LO noise. LO noise is independent of sampling. In this paper, we focus on $\mathrm{LO}$ noise.

Intrinsic and external RF noise can be analyzed in frequency domain because the sampling mixer can be approximated by a linear time-invariant system from the RF port to the output port. This is not true for LO noise because the relation from LO to output is not linear. In addition, the system has two input ports, so from any one of the input ports to the output port, the system must be considered time-varying. It is possible to perform noise simulation for periodically time-varying systems using two-dimensional impulse responses [7]. However, it is difficult to obtain analytical expression using this method, and the approach is only applicable to systems with small excitation and therefore ill-suited for analyzing LO noise in the sampling mixer. Traditionally, it has been argued that small signal $g_{m}$ from LO to output is small

$$
g_{m}=\frac{\partial I_{D}}{\partial V_{G}}=K V_{\mathrm{DS}}
$$

since $V_{\mathrm{DS}}$ is small, so the amount of noise coupled from LO to output is also small. This argument is not rigorous because $g_{m}$ changes with time. Therefore, to deal with this situation properly, a time-domain method based on SDE is required.

\section{ANALYSIS OF LO NOISE USING SDE's}

\section{A.. Derivation of Noise Variance Process}

We now formulate the differential equation governing the circuit in Fig. 1. The MOS transistor works in the triode region when the gate voltage is high. Kirchhoff's current law on the output node gives

$$
K\left(V_{\mathrm{GS}}-V_{t}\right) V_{\mathrm{DS}}-\frac{K}{2} V_{\mathrm{DS}}^{2}=C \frac{d V_{D}}{d t}
$$

where $K=\mu C_{\mathrm{ox}} W / L$ is the device constant, $V_{t}$ is the MOS threshold voltage, $C$ is the sampling capacitor, and $V_{D}, V_{S}$, and $V_{G}$ are drain, source, and gate voltages, respectively. Here, the input is taken as source, and the output as drain. This is a nonlinear equation, but as mentioned before, the nonlinear terms are small by design, so we only need to work with its linear part

$$
K\left(V_{G}-V_{t}\right) V_{D}+C \frac{d V_{D}}{d t}=K\left(V_{G}-V_{t}\right) V_{S}
$$

We are interested in the case where $V_{S}$ is deterministic and where gate voltage contains the deterministic signal $V_{G}$, plus a noise term $\sigma \xi_{t}$

$$
K\left(V_{G}+\sigma \xi_{t}-V_{t}\right) V_{D}+C \frac{d V_{D}}{d t}=K\left(V_{G}+\sigma \xi_{t}-V_{t}\right) V_{S} .
$$

Noise is modeled as white noise because we are interested in the effect of thermal noise at the gate. White noise is also chosen for its mathematical tractability. White noise has the property that each sample is Gaussian distributed (with infinite variance), and samples from different time points are uncorrelated.

To write the above equation in the standard SDE form, we formally multiply both sides by $d t$, and replace white noise $\xi_{t} d t$ by its formal integral $d W_{t}$. As a convention in SDE literature, we rewrite the output process $V_{D}$ as $X_{t}$, and the input process $V_{S}$ as $u_{t}$. Finally, we define MOS conductance $g_{t}=K\left(V_{G}-V_{t}\right)$, which can be a function of time, and get

$$
d X_{t}=\frac{g_{t}}{C}\left(u_{t}-X_{t}\right) d t+\frac{K}{C}\left(u_{t}-X_{t}\right) \sigma d W_{t} .
$$

The first term on the right-hand side is the deterministic part, which describes the trajectory of the output process without noise. The second term is the stochastic part, which modifies the trajectory due to noise. This is a first-order SDE in the standard form, whose solution is completely known [8]. Our aim is to extract information such as $E\left[X_{t}\right]$ and $E\left[X_{t}^{2}\right]$ from (8). We highlight the results below. The mathematical background and detailed derivation are presented in the Appendix.

First, we formally integrate both sides of (8), then take expectation

$$
\begin{aligned}
E\left[X_{t}\right]=E\left[X_{0}\right]+E\left[\int_{0}^{t} \frac{g_{t}}{C}\left(u_{s}-X_{s}\right) d s\right. & \\
& \left.+\int_{0}^{t} \frac{K}{C}\left(u_{s}-X_{s}\right) \sigma d W_{s}\right] .
\end{aligned}
$$

The second integral is a stochastic integral with respect to Wiener process whose expectation is 0 for all $t$ as noted (21) in the appendix. In the differential form

$$
\frac{d}{d t} E\left[X_{t}\right]+\frac{g_{t}}{C} E\left[X_{t}\right]=\frac{g_{t}}{C} u_{t}
$$

This is the differential equation describing the mean of the output process, which happens to be exactly the same as the differential equation for the system without noise.

Next, we evaluate $E\left[X_{t}^{2}\right]$, which requires the full power of stochastic calculus. As will be shown in the appendix, the equation governing the expected value of the square of the process turns out to be

$$
\begin{aligned}
E\left[X_{t}^{2}\right]=E\left[X_{0}^{2}\right]+\int_{0}^{t} & \frac{2 g_{t}}{C}\left(E\left[X_{s}\right] u_{s}-E\left[X_{s}^{2}\right]\right) d s \\
& +\frac{\sigma^{2} K^{2}}{C^{2}} \int_{0}^{t} E\left[\left(u_{s}-X_{s}\right)^{2}\right] d s
\end{aligned}
$$

If we now define the output noise to be $N_{t}=X_{t}-E\left[X_{t}\right]$, then combine (11) with (10) and put the result into differential form, we have

$$
\frac{d}{d t} E\left[N_{t}^{2}\right]+\left(\frac{2 g_{t}}{C}-\frac{\sigma^{2} K^{2}}{C^{2}}\right) E\left[N_{t}^{2}\right]=\frac{\sigma^{2} K^{2}}{C^{2}}\left(E\left[X_{t}\right]-u_{t}\right)^{2} .
$$


Equations (10) and (12) are the ordinary differential equations (ODE's) which describe the mean and the variance of the output process in the presence of noise at the gate. At this point, we have turned an SDE into an ordinary differential equation which, in this case, also happens to be first-order linear time varying, for which an analytical solution exists. From this point on, only knowledge of ODE's is necessary. $E\left[N_{t}^{2}\right]$ contains all we need to know about the second-order statistics of the output noise variance. Since output is taken in the sampled data domain and the time constant associated with the low-pass filter formed by the MOS and the capacitor is small, consecutive sample points are not correlated. Also note that since noise amplitude is assumed to be small, i.e., $2 g_{t} / C \gg \sigma^{2} K^{2} / C^{2}, \sigma^{2} K^{2} / C^{2}$ term can be neglected in (12).

Equation (12) can be derived in a number of different ways. For example, without the $\sigma^{2} K^{2} / C^{2}$ term, (12) can be derived using the linearization approach in [4], where the trajectory without noise is first solved, and noise is modeled as small signal perturbation about the deterministic trajectory. This approach assumes that the modulation of the noise source by the noise part of $X_{t}$ is small. For sufficient simple systems such as ours, it is actually possible to solve the SDE directly without the linearization step. This is the approach presented in the Appendix. This approach does not work in the full generality of [4] (for example, it cannot capture the nonlinearity in the transistor model), but it quantifies the noise-noise modulation effect, which is the $\sigma^{2} K^{2} / C^{2}$ term, whose effect is indeed small. Further, because our system is sufficiently simple, (12) can be solved in closed form. This closed-form solution would allow us to approach the problem from an analytical view point, from which design intuition may be gained.

\section{B. Solution of Noise Variance}

We proceed to solve (12). First consider the case where $g_{t}$ is constant, i.e., there is no switching. We assume that $u_{t}$ is a deterministic sinusoidal $A \cdot \cos (\omega t)$, so $E\left[X_{t}\right]$ is also a sinusoidal with a gain and a phase shift as in (10). Assuming a small system time constant from (10), we know that the gain is close to one, and the phase shift is $\omega C / g$. This allows us to work out the right-hand side of (12), which is a sinusoid with twice the input frequency plus a dc term. Then, by solving (12), we are able to conclude that the noise variance is periodically time varying with of the input frequency. The dc term of the variance is

$$
E\left[N_{t}^{2}\right]=\frac{A^{2}}{4} \cdot \frac{\omega^{2} C}{K\left(V_{G}-V_{t}\right)^{3}} \cdot \sigma^{2} .
$$

This is the variance of the output due to a noisy LO when the deterministic part of LO is constant. The solution also applies to the case where switching $\mathrm{LO}$ voltage has zero fall time. Comparing to $k T / C$, the magnitude of noise variance is actually much smaller, and for practical purposes, it can be ignored.
Next we consider the case where there is switching. In particular, we are interested in the case when the LO voltage has a finite fall time. Fall time is technology limited, and it can be much larger than the filter time constant. So essentially, we have the case where $g_{t}$ decreases linearly to zero prior to cutoff, i.e., $g_{t}=-\alpha t$, where $\alpha=K V_{G} / T_{f}$, as in Fig. 1 . To solve for noise variance in this case, we recognize that (10) and (12) are linear ODE's, whose solutions are well known, as shown in (14), at the bottom of the page, where for convenience, we defined $\beta^{2}=\alpha / 2 C$. The explicit evaluation of this integral is difficult for a sinusoidal $u(t)$, however, the integral can be simplified if we recognize that filter time constant is small, so only points within a few time constants are of interest, and hence the sinusoidal $u(t)$ can be approximated by its Taylor series right before the time of cutoff. The key observation is that we may solve the equation for each sample and interpret the result in the sampled data domain.

Let the sample time be 0 , and let the sinusoidal input be

$$
u(t)=A \cdot \cos \left(\omega\left(t-t_{0}\right)\right) .
$$

Define $u_{0}=A \cdot \cos \left(\omega t_{0}\right)$, i.e. the input signal level at time 0 , then the Taylor series approximation of $u(t)$ is

$$
u(t)=u_{0}+\sqrt{1-u_{0}^{2}} \cdot \omega t .
$$

It is now possible to evaluate (14) by substituting (15). The evaluation is laborious, and it involves numerical evaluation of error integrals. Fortunately, we only need to solve for $E\left[N_{0}^{2}\right]$ since cutoff occurs at time 0 and we are only interested in sampled data. It turns out that the final expression is

$$
E\left[N_{0}^{2}\right]=\left(A^{2}-u_{0}^{2}\right) \sqrt{\frac{K}{C}}\left(\frac{T_{f}}{V_{G}}\right)^{3 / 2} \omega^{2} \cdot \sigma^{2} \cdot 0.443
$$

where the numerical constant comes from the evaluation of a definite integral. We observe that noise variance is frequency dependent. Noise variance also depends on $u_{0}$, therefore it is time varying, with its maximum achieved when the input crosses zero. In other words, (16) gives a bound on noise variance when $u_{0}$ is set of 0 . Again, the noise power calculated above is independent of sampling. In the subsampling case, noise power for each sample is the same, but more noise is folded into the signal band, as in the case for intrinsic noise.

We will work out a numerical example that compares the effects of noise from various noise sources. At low frequency, intrinsic $k T / C$ noise is much larger than $\mathrm{LO}$ noise. However, as frequency goes higher, LO noise due to finite fall time becomes significant. If the noise power at $\mathrm{LO}$ has equivalent resistance equal to $500 \Omega$, take the typical values for $V_{G}=$ $2.5 \mathrm{~V}, A=0.5 \mathrm{~V}, C=0.4 \mathrm{pF}, T_{f}=200 \mathrm{ps}$, filter time constant $\tau=10 \mathrm{ps}$, and LO noise power becomes comparable to $k T / C$ at about $2 \mathrm{GHz}$. In mixer design, jitter noise must also be taken into consideration. If we assume a typical phase noise figure for the oscillator at $-120 \mathrm{dBc}$, one channel away

$$
E\left[N_{t}^{2}\right]=\frac{\sigma^{2} K^{2}}{C^{2}} e^{2 \beta^{2} t^{2}} \int_{-\infty}^{t} e^{-2 \beta^{2} \tau_{1}^{2}}\left(e^{\beta^{2} \tau_{1}^{2}} \int_{-\infty}^{\tau_{1}} 2 \beta^{2} \tau_{2} \times e^{-\beta^{2} \tau_{2}^{2}} u\left(\tau_{2}\right) d \tau_{2}+u\left(\tau_{1}\right)\right)^{2} d \tau_{1}
$$


TABLE I

COMPARISON Among InTRINSIC, LO, AND JitTER NoISE

\begin{tabular}{c|c|c|c}
\hline $\begin{array}{c}\text { Input } \\
\text { Frequency }\end{array}$ & $\begin{array}{c}\text { Intrinsic } \\
\text { noise power }\left(V^{2}\right)\end{array}$ & $\begin{array}{c}\text { LO } \\
\text { noise power }\left(V^{2}\right)\end{array}$ & $\begin{array}{c}\text { Jitter } \\
\text { noise power }\left(V^{2}\right)\end{array}$ \\
\hline $200 \mathrm{MH} /$ & $1 \mathrm{e}-8$ & $1.36 \mathrm{e}-10$ & $7.2 \mathrm{e}-8$ \\
\hline $2 \mathrm{GHz}$ & $1 \mathrm{e}-8$ & $1.36 \mathrm{e}-8$ & $7.2 \mathrm{e}-9$ \\
\hline
\end{tabular}

from the center frequency with channel width $1.7 \mathrm{MHz}$, and take LO frequency to be very close to the input frequency, jitter noise power is found to be comparable to intrinsic or LO noise at high frequency [5]. Here, we have not taken the spectral characteristics of jitter noise into consideration because this necessarily depends on the design of the oscillator. Table I summarizes the numerical example.

\section{Discussion on Time-Varying Noise}

In the sampled data domain, variance of the LO noise varies with time at twice the input frequency; so, LO noise is cyclostationary. We can think of it as a low-pass noise modulated by $\cos (\omega t)$. Because LO noise is not wide-sense stationary, it does not make sense to talk about its power spectral density. In fact, the time-varying property is intrinsic to the noise process because the time-varying frequency is the same as the signal frequency; hence, there is no way to filter out the time-varying part without also filtering out the signal itself at the same time.

Because LO noise is not stationary, it is not possible to determine the amount of noise that falls in the signal band. Nevertheless, we may visualize time-varying noise by looking at its sample paths. Because each sample path is modulated by $\cos (\omega t)$, the noise spectrum is shifted by $\omega$, but still maintains the low-pass shape. In this case, after noise folding due to sampling, the output spectrum is approximately white.

For time-varying LO noise, we also see that the noise power is maximum at zero-crossing, which we can regard as an upper bound. This bound is tight for applications where frequency or phase modulation schemes are used (e.g., QPSK). This is because in such modulation schemes, information is carried precisely at the zero-crossing points.

It should be stressed that the above conclusions are drawn with the simplest MOS model, without taking into account many of the second-order effects. A full transistor model would involve solving differential equations higher than first order, for which a closed-form solution is difficult to obtain. However, we expect the first-order model to capture the major effects.

\section{CONCLUSION}

Noise in a sampling mixer is analyzed. Noise due to external RF and intrinsic sources are analyzed using frequency domain methods. LO noise is characterized by solving a SDE analytically in time domain. The closed-form solution is obtained, which can be used to guide the design process. It is shown that LO noise becomes comparable to other noise sources at high frequencies, and when the LO voltage has a large fall time. This effect is in addition to LO jitter, which has to be combined with noise analysis to obtain a complete picture of sampling mixer noise.

\section{APPENDIX \\ DeRIVATION OF $E\left[X_{t}^{2}\right]$ IN THE SDE}

In this Appendix, we will derive (11). The necessary background is introduced to make this section as self-contained as possible.

Thermal noise is often modeled as white noise (denoted as $\xi_{t}$ ), whose power spectral density is flat across all frequencies up to infinity. However, white noise is not a physical process because it has infinite power. To treat white noise rigorously, we need to define its integral, called a Wiener process, which can be approximated by physical processes

$$
W_{t}=\int_{0}^{t} \xi_{s} d s
$$

A Wiener process has a continuous sample path and independent Gaussian increments. However, sample paths of a Wiener process have unbounded variation (or infinite length), so it is difficult to define integration with respect to a Wiener process. Ito's stochastic calculus is invented precisely to solve this problem [8]-[10].

Central to Ito's theory is a characterization of the variance of a Wiener process. The quadratic variation process, denoted by $\langle W\rangle_{t}$, can be thought of as the limit

$$
\langle W\rangle_{t}=\lim \sum_{i=1}^{n}\left(W_{t_{i}}-W_{t_{i-1}}\right)^{2} .
$$

For a Wiener process $\langle W\rangle_{t}=t$.

In the study of SDE's such as

$$
d X_{t}=a\left(X_{t}, t\right) d t+b\left(X_{t}, t\right) d W_{t}
$$

we need to deal with stochastic integrals. If we formally integrate the SDE, the right-hand side consists of two types of integrals. The integral with respect to time is straightforward because it can be defined for each sample path. The integral with respect to a Wiener process $W_{t}$ is more problematic, for which it is necessary to go back to the Riemann-Stieltjes definition

$$
\int_{0}^{t} f\left(W_{s}, s\right) d W_{s}=\lim \sum_{i=0}^{n-1} f\left(W_{t_{i}}, t_{i}\right)\left(W_{t_{i+1}}-W_{t_{i}}\right)
$$

Note here that the intermediate point in the Riemann sum is chosen to be the first end point of the interval. The calculus developed from the approximating sum (20) is said to be a stochastic integral in Ito's sense.

Stochastic integrals have a number of properties. First, notice that

$$
E\left[\int_{0}^{t} f\left(W_{s}, s\right) d W_{s}\right]=0
$$


Also, the integral has unbounded variation, so it, too, has a quadratic variation process

$$
\begin{aligned}
\left\langle\int_{0}^{t} f\left(W_{s}, s\right) d W_{s}\right\rangle_{t} & =\int_{0}^{t} f^{2}\left(W_{s}, s\right) d\langle W\rangle_{s} \\
& =\int_{0}^{t} f^{2}\left(W_{s}, s\right) d s
\end{aligned}
$$

where, in the second equality, we used (18). A slight generalization of the above equation can actually be taken as the definition of stochastic integration.

This definition of stochastic integral requires the following modification to the fundamental theorem of calculus

$$
\phi\left(X_{t}\right)=\phi\left(X_{0}\right)+\int_{0}^{t} \phi^{\prime}\left(X_{s}\right) d X_{s}+\frac{1}{2} \int_{0}^{t} \phi^{\prime \prime}\left(X_{s}\right) d\langle X\rangle_{s}
$$

where $\phi(x)$ is any twice-differentiable function with continuous second derivatives. The above equation is known as Ito's formula, and is a fundamental tool in stochastic calculus. Ito's formula allows us to manipulate nonlinear transformations of stochastic processes. Because of the last correction term which involves the quadratic variation process, normal rules of solving differential equations need to be modified.

We now use stochastic calculus to solve for $E\left[X_{t}^{2}\right]$ in (8)

$$
d X_{t}=\frac{g_{t}}{C}\left(u_{t}-X_{t}\right) d t+\frac{K}{C}\left(u_{t}-X_{t}\right) \sigma d W_{t} .
$$

The evaluation of $E\left[X_{t}^{2}\right]$ requires Ito's formula (23), where we set $\phi(x)=x^{2}$

$$
X_{t}^{2}=X_{0}^{2}+\int_{0}^{t} 2 X_{s} d X_{s}+\langle X\rangle_{t} .
$$

We need to work out the quadratic variation process $\langle X\rangle_{t}$. If we write out $X_{t}$ explicitly by formally integrating (24)

$$
X_{t}=\int_{0}^{t} \frac{g_{t}}{C}\left(u_{s}-X_{s}\right) d s+\int_{0}^{t} \frac{K}{C}\left(u_{s}-X_{s}\right) \sigma d W_{s}
$$

we see that $X_{t}$ consists of two parts. The time integral is of bounded variation, so it does not contribute to the quadratic variation of $X_{t}$. The second integral is with respect to a Wiener process, and by (22)

$$
\begin{aligned}
\langle X\rangle_{t} & =\left\langle\int_{0}^{t} \frac{K}{C}\left(u_{s}-X_{s}\right) \sigma d W_{s}\right\rangle_{t} \\
& =\frac{\sigma^{2} K^{2}}{C^{2}} \int_{0}^{t}\left(u_{s}-X_{s}\right)^{2} d s .
\end{aligned}
$$

Now, substitute (24) and (27) into (25), take expectation on both side, recognize the fact that the expectation of the stochastic integral of $X_{t}$ with respect to Wiener process is zero, i.e., (21), we have

$$
\begin{aligned}
E\left[X_{t}^{2}\right]=E\left[X_{0}^{2}\right]+\int_{0}^{t} \frac{2 g_{t}}{C}\left(E\left[X_{s}\right] u_{s}-E\left[X_{s}^{2}\right]\right) d s \\
+\frac{\sigma^{2} K^{2}}{C^{2}} \int_{0}^{t} E\left[\left(u_{s}-X_{s}\right)^{2}\right] d s
\end{aligned}
$$

which is (11).

\section{ACKNOWLEDGMENT}

The authors wish to thank Prof. A. Heunis for many helpful discussions on the mathematical subtleties of stochastic calculus.

\section{REFERENCES}

[1] A. Hairapetian, "An 81-MHz IF receiver in CMOS," IEEE J. Solid-State Circuits, vol. 31, pp. 1981-1986, Dec. 1996.

[2] P. Y. Chen, A. Rofougaran, K. A. Ahmed, and A. A. Abidi, "A highly linear 1-GHz CMOS downconversion mixer," in Proc. Eur. Solid-State Circuits Conf., Sevilla, Spain, 1993, pp. 226-227.

[3] S. Sen, "Design of sampling mixer and A/D converter for high IF digitization," Ph.D. dissertation, Univ. Waterloo, ON, Canada, 1997.

[4] A. Demir, E. W. Y. Liu, and L. S.-Vincentelli, "Time-domain non-Monte Carlo noise simulation for nonlinear dynamic circuits with arbitrary excitations," IEEE Trans. Computer-Aided Design, vol. 15, pp. 493-505, May 1996.

[5] T. C. Weigandt, B. Kim, and P. R. Gray, "Analysis of timing jitter in CMOS ring oscilllators," in Proc. Int. Symp. Circuits and Systems, 1994, vol. 4, pp. 27-30.

[6] D. Shen, C.-M. Hwang, B. B. Lusignan, and B. A. Wooley, "A 900-MHz RF front-end with integrated discrete-time filtering," IEEE J. Solid-State Circuits, vol. 31, pp. 1945-1996, Dec. 1996.

[7] C. D. Hull, "Analysis and optimization of monolithic RF downconversion receivers," Ph.D. disserttion, Univ. California at Berkeley, 1992.

[8] I. Karatzas and S. E. Shreve, Brownian Motion and Stochastic Calculus, 2nd Edition. New York: Springer-Verlag, 1991.

[9] A. Heunis, "Stochastic calculus," STAT 902 Course Notes, Univ. Waterloo, ON, Canada, 1996.

[10] Z. Schuss, Theory and Applications of Stochastic Differential Equations. New York: Wiley, 1980.

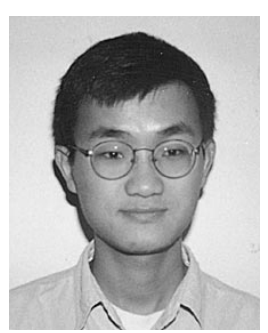

Wei Yu received a joint B.S. degree in computer engineering and mathematics from the University of Waterloo, ON, Canada, in 1997, and the M.S. degree in electrical engineering from Stanford University, CA, in 1998. He is currently working toward the Ph.D. degree in the Information Systems Laboratory of the Electrical Engineering Department, Stanford University.

His current research interest is in digital communications.

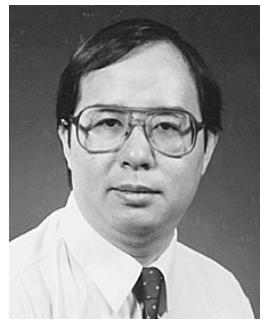

Bosco H. Leung (S'84-M'85-SM'92) received the B.Sc. degree from Rensselaer Polytechnic Institute, Troy, NY, in 1979, the M.Sc. degree from the California Institute of Technology, Pasadena, in 1980, and the Ph.D. degree from the University of California, Berkeley, in 1987, all in electrical engineering.

From 1980 to 1983, he was with Northern Telecom, Canada, working on analog circuit design $\mathrm{He}$ is currently a Professor with the Electrical and Computer Engineering Department, University of Waterloo, ON, Canada. His main research interest is in CMOS and BiCMOS mixed A/D integrated circuits, in particular on analog-to-digital converters. $\mathrm{He}$ has published over 30 technical papers and has received patents in this area. $\mathrm{He}$ has recently developed an ultra low-power low-distortion mixer and A/D converters for direct conversion based wireless receivers, and is also working on frequency synthesizers and other front-end designs for wireless receivers.

Dr. Leung is currently an Associate Editor with the IEEE TRANSACTIONS on Circuits AND Systems II. 